\title{
An IC yield enhancement approach by ARMA modeling and dynamic process control
}

\author{
Liren Yan • Wei Huang
}

Received: 27 October 2007 / Accepted: 20 June 2008 /Published online: 30 July 2008

(C) The Author(s) 2008

\begin{abstract}
The IC industry has been growing rapidly in the past decades. The continuous scaling-down of the feature size requires IC machines of highest performances, and pushes the IC manufacture to its utmost technology limits. Nowadays, IC manufacturers employ tightly fixed process parameters as their strategy to improve the yield. In this paper, a "softer" way is proved to be more potential in further improving and managing the yield of IC products. A novel concept which suggests running an IC procedure with dynamic process parameters is explained in detail. By using auto-regressive moving-average (ARMA) model, effects of the key process steps can be predicted and the related process parameters can be adjusted self-reactively. Since the discussion indicates distinct improvements of the IC yields, it seems the time to break the rule of "using stationary process parameters".
\end{abstract}

Keywords Advanced process control - Dynamic process parameters $\cdot$ IC yield enhancement $\cdot$ Self-adapt modification

\section{Introduction}

Moore's law has been adopted as a yardstick by the IC industry for more than 40 years. Driven by the law, the IC products are distinctly improved in speed, integrated density, energy consumption, and overall performances. On the other hand, as the technology node approaches $45 \mathrm{~nm}$ and beyond, the manufacturing cost is soaring and becomes more and more prohibitive. Among all factors to the total manufacturing cost, the IC yield has been proved one of the most important [1].

L. Yan $(\bowtie) \cdot$ W. Huang

Institute of Microelectronics, Tsinghua University,

Beijing, People's Republic of China

e-mail: ylr@tsinghua.edu.cn
Each phase in the IC process flow, from original design to final packaging, impacts the yield as well as the reliability of the product. Statistical methods such as data mining approaching with classification/regression trees have been used to identify key factors that limit IC yields [2-4]. Accordingly corrective activities can be determined to improve the IC yields.

The yield enhancement methods, in general, can be grouped into three categories.

1) Design for manufacturing (DFM). In this method, every step involving equipments, raw materials, environmental conditions, process steps, and settings is carefully studied to improve efficiency and reduce interference which limits throughput, cycle time, and wafer-test yields [5-7]. Ideally, if all phases are optimized after DFM analyses, high yield of IC products can be ensured.

2) Advanced quality management. Quite a few advanced quality control methods, such as total quality management (TQM) and six-sigma approach, have been utilized in semiconductor manufacturing to eliminate product defects and shorten cycle time [8-11]. Some other methods, such as recipe control and management system (RCMS) focused on escaping low yield due to human errors [12], and smart dummy/test wafer control focused on both improving yield and cutting down manufacturing cost [13-14], have also been developed.

3) Advanced process control (APC). Typically an IC process involves hundreds of steps. APC methods are used to improve qualities of those key process steps, such as implantation [15], photolithography [16], and plasma etching [17], etc. Some IC process steps are so complicated that they are dealt with by neural networks in recent works [17-19].

If a process has been well controlled and high yield is achieved already, the run to run and wafer to wafer 
consistency becomes the most critical issue. When a new fabricator is built, the process conditions for each step and the system configuration will be set up identical to the previous one, so that the investigation on yield does not have to be repeated [5, 20]. Furthermore, the process parameters/ conditions of a step are fixed strictly based on the assumption that the process results can be exactly repeated under fixed settings. In this paper, we proposed that self-reacting dynamic process parameters leads to equally stable and potentially better production.

The stable run to run yield control of a modern process is very difficult to achieve due to the large numbers of variables/ parameters that are available for operator to manipulate. Some works utilize neural networks to deal with the complexities [16-18]. In this paper, we ascribe the run to run variation of a process step to only one factor, the time. The possible fluctuation can be forecast by auto-regressive moving-average (ARMA) models, which are based on the time series analysis. According to the predictions, the fluctuation can be compensated by modifying process parameters thus the process stability and the yield are maintained by such a dynamic process control method.

In Section 2, an overview on ARMA model is presented. The basic idea of time series analysis is also introduced. In Section 3, we apply the ARMA model to control process steps. Cases involving one or more time series are studied. In Section 4, the dynamic process control method is applied on a Si/SiGe epitaxial process. And in Section 5, we conclude our work on this yield enhancement approach.

\section{The ARMA model}

The ARMA model was presented by American statistician George EP Box and British statistician Gwilym M Jankins in 1968, which is also known as the B-J model. As an accurate short-term predicting arithmetic, the ARMA model is one of the most widely used time series analysis models applied to industry and economy analyzing/predicting [21].

A time series is a set of observed data in sequence. Although the data for the next time is unknown, it can be predicted statistically by a time series model. In most cases, the model can be expressed as a summation of items either related to the observed data or the residuals, i.e.:

$$
\begin{aligned}
x_{t}= & \phi_{1} x_{t-1}+\phi_{2} x_{t-2}+\ldots+\phi_{p} x_{t-p}+\varepsilon_{t}+\theta_{1} \varepsilon_{t-1} \\
& +\theta_{2} \varepsilon_{t-2}+\ldots+\theta_{q} \varepsilon_{t-q}
\end{aligned}
$$

In the formula, $x_{t}$ is the sample value at time $t$, and $x_{t-i}$ refers to time $t-i, \varepsilon_{t-j}$ is the residual at time $t-j$, and $\phi_{i}, \theta_{j}$ are weights which can be fit later in the "parameter estimation" step.
In (1), $p$ is called the order of "auto-regressive" part, and $q$ the order of "moving average" part. Thus, (1) is named the $\operatorname{ARMA}(p, q)$ model [22]. Once the values of $p, q$, and the weights are determined, the data for the next time can be estimated accurately by using this model.

An ARMA is a combination of AR and MA models. While $q=0$, the $\operatorname{ARMA}(p, q)$ becomes a pure auto-regressive model $\operatorname{AR}(p)$, while $p=0$, it becomes a pure moving-average model $\mathrm{MA}(q)$.

For a particular time series, its ARMA model can be established by following the procedures shown in Fig. 1.

One prerequisites of ARMA modeling is that the time series must be at least a weak stationary stochastic series if not stationary. Many stochastic time series in practice are not stationary, which may lead to invalid regressions. Therefore, stationarity of a time series must be tested before ARMA modeling and non-stationary time series must be stationarized. The autocorrelation value can be used as the criteria to identify the stationarity. If most of the values are out of confidence interval in the correlogram, and don't converge to zero, the time series is non-stationary. Another commonly used method is the unit root test. If one unit root exists, the time series is not stationary, but it is a 1st-order differential stationary series. If there are $n$ unit roots, the time series is $n$ th-order differential stationary. The commonly used methods to stationarize a time series include differential transform, and/or logarithm transform.

Orders, i.e., the values of $p$ and $q$ of an $\operatorname{ARMA}(p, q)$ model, are determined in the model identification step in Fig. 1. These orders can be identified via diagrams of autocorrelation and partial correlation of the time series. If the $q$ th-order autocorrelation coefficient becomes very small, and

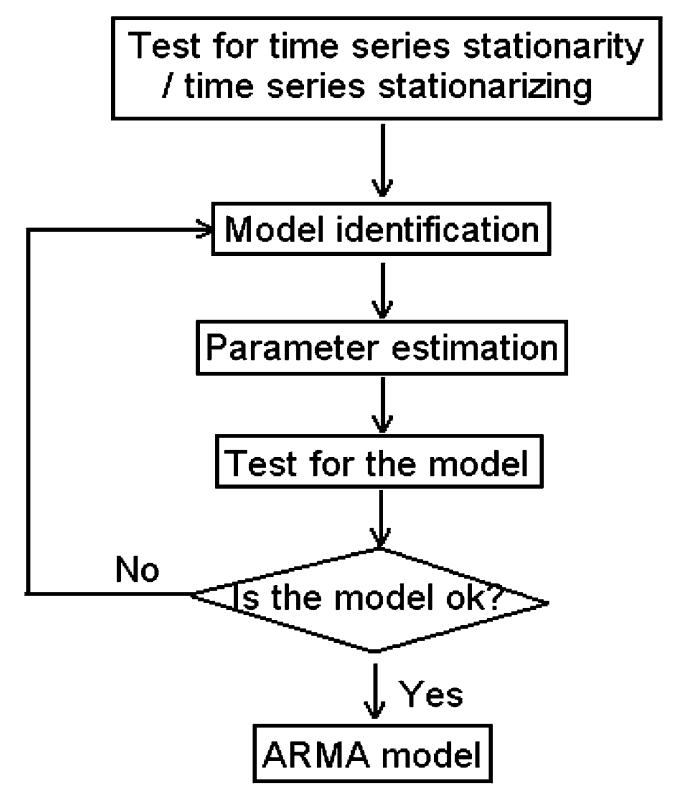

Fig. 1 Steps of establishing the ARMA model of a time series 
the auto-correlogram is bob-tailed from there, the model must be an $\operatorname{MA}(q)$. Similarly if the partial correlogram becomes bob-tailed at the $p$ th-order, the desired model should be an $\operatorname{AR}(p)$. When both the auto-correlogram and the partial correlogram are long-tailed, the model can not be an $\operatorname{MA}(q)$ or an $\operatorname{AR}(p)$, but an $\operatorname{ARMA}(p, q)$. These general criteria are listed in Table 1.

It is hard to determine the $p$ and $q$ orders of an $\operatorname{ARMA}(p$, $q$ ) model directly. Regularly, a pair of guess values of $p$ and $q$ is estimated to establish an ARMA model. After calculating all the weights in formula (1), the model is tested for. After several repetitions, the optimized ARMA model for a specific time series can finally be obtained as shown in Fig. 1.

Having determined $p$ and $q$, the coefficients in Eq. (1) are fit with the observed data. The model is tested based on the rule that the residuals at different time, $\varepsilon_{t}$, should form a white noise series which is often identified by $Q$ testing for the residual auto correlations, as well as by observing autocorrelation and partial autocorrelation coefficients of the residuals. If the residuals failed to form a white noise series, the model has to be re-established. On the other hand, if more than one ARMA models are tested to be valid, the most accurate one can be determined if one calculate the standard deviations of the dependent variables from the models, and compare these deviations. For these models, the Akaike info criterion or Schwarz Criterion values can also be compared. Generally, the smaller the value is, the more accurate the model is indicated.

\section{Prediction and the dynamic process control}

In this section, we will discuss how to apply the time series analysis in advanced IC process control.

The application won't be a direct one because of the strict requirement that a time series under processing must be at least weak stationary. For instance, in practice, the film thickness is always measured after depositing a film layer. It is possible to forecast film thickness of the next deposition by ARMA modeling, and then adjust the process conditions so the resulted film thickness could meet the specification. Although the "forecast-correction" scheme may work at the first time, it is unlikely that such a scheme may work all the time. Because once one has changed the process conditions, the time series of film thickness is disturbed, and possibly becomes non-stationary.
To overcome the problem, IC process details are taken into consideration. Normally, the data of a process step depend on more than one process variables. These variables can be roughly divided into two groups. One group varies from time to time, and can be predicted by time series analysis. The other is to be adjusted so that the variance of the first group can be compensated accordingly. The former is used only for prediction, and the latter is used only for correction. In this way, one or more variables are selected, and their values at different time form the time series. Since these variables keep undisturbed in the correction phase, their time series may keep stationary.

\subsection{One time series case}

In this part, we take the epitaxy film thickness for instance to show our dynamic process control idea.

Epitaxy is an important process in semiconductor manufacturing which is widely used for growing thin-film of varieties of materials. A critical requirement for this unit process is that the film thickness must be uniform not only at different sample points on one wafer, but also on different wafers, and wafers of different batches.

Each time when an epitaxy process finishes, film thickness and some process parameters are measured, or calculated from measured data. One of the parameters is chosen and its values at different times form a time series. Based on this time series, the process parameter for the next depositing can be forecast by an ARMA model.

In order to not only meet the requirement on film thickness uniformity but also keep the time series stationary, we prefer adjusting other process parameters. This implies we must know the relationships between film thickness and the process parameters. For the epitaxy process, a simple process model can be used:

$D_{t}=S_{t} \cdot T_{t}$

According to the model, the thickness $D$ is only determined by the epitaxial growing speed $S$, and the processing time T. Film thickness at each deposition can be calculated as the multiply of these two parameters.

In nature, the epitaxial conditions such as gas flows, temperature, pressure, etc. can not be controlled absolutely steady. As a result, thickness of the films varies randomly. In our simple epitaxial model, fluctuation of these process conditions is reduced to the fluctuation of the growing speed.
Table 1 Model selection criteria

\begin{tabular}{llll}
\hline Model & AR $(p)$ & MA $(q)$ & ARMA $(p, q)$ \\
\hline Auto-correlogram & Long-tailed & Bob-tailed at $q$ th order & Long-tailed \\
Partial auto-correlogram & Bob-tailed at $p$ th order & Long-tailed & Long-tailed \\
\hline
\end{tabular}


Each time the film thickness is measured, the growing speed for that time is calculated, and its values at different times form a time series. The ARMA method can be successfully used to forecast the growing speed for the next time. If by prediction, the film growing speed will increase, the process time will be shortened by an amount calculated accordingly, and vice versa. In this way, a better thickness control is achieved.

In this section, we have studied on how to dynamically control a process step which can be described by a simple process model. In the next section, we will discuss the case of more complicated process model, where two or more time series are involved.

\subsection{More than one time series case}

Most IC processes are described by more complicated process models. To dynamically control these processes, two or more related time series must be considered. As an example, oxidation process is discussed here.

The film thickness after a Si oxidation process is described by (3), the well known Deal-Grove model [23].

$x^{2}+A x=B(t+\tau)$

where $x$ is the thickness of $\mathrm{SiO}_{2}$ film, $t$ is the process time, $A$ and $B$ are the coefficients, and $\tau$ is a time constant. To simplify the problem, we assume $\tau$ to be 0 which does not affect our conclusion.

The values of $t$ and $x$ are recorded at each time as the known data, i.e. $t_{i}$ and $x_{i}$. According to (3), since the target thickness is known, given the $A_{i+1}$ and $B_{i+1}$ for the next oxidation by prediction, $t_{i+1}$ can be corrected, so that $x_{i+1}$ can meet the expectation. For the oxidation process, the "forecast-correction" scheme works under the requirement to deal with two time series.

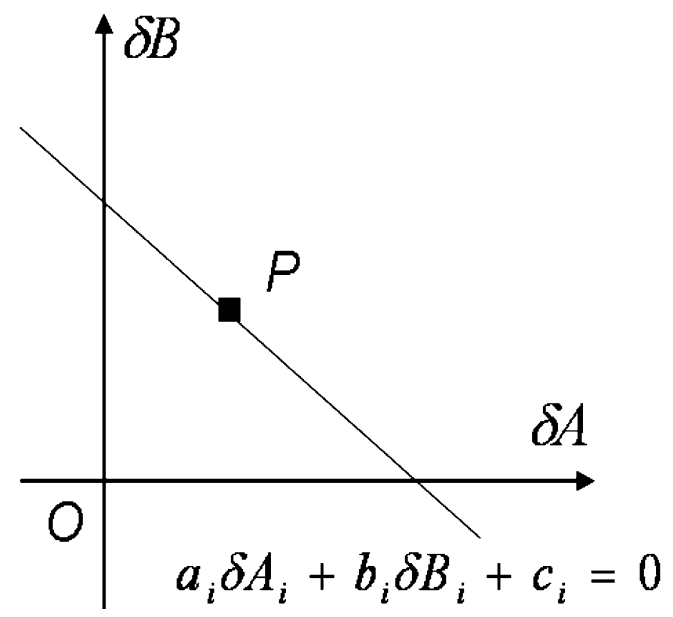

Fig. 2 The point on the line and nearest to origin

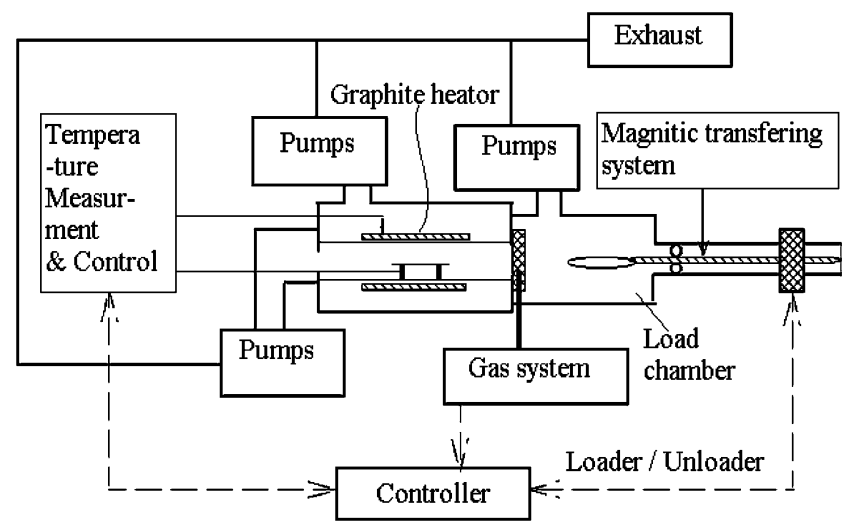

Fig. 3 The self-made UHV/CVD SiGe epitaxial system

Statisticians haven't told us how to predict two variables at a same time. The following steps are suggested to solve this problem.

1) From formula (3) and the known $x_{i}, t_{i}(i=1,2, \ldots, n)$ values, we can get:

$$
a_{i}^{\prime} A_{i}+b_{i}^{\prime} B_{i}+c_{i}^{\prime}=0 \quad i=1,2, \ldots n
$$

where $a_{i}^{\prime}=x_{i}, b_{i}^{\prime}=-t_{i}, c_{i}^{\prime}=x_{i}^{2}$. The value $a_{i}^{\prime}, b_{i}^{\prime}, c_{i}^{\prime}$ are known, and $A_{i}, B_{i}$ are variables.

2) $A_{i}$ and $B_{i}$ depend on oxidation conditions, such as gas flows, temperature, pressure, etc. These values vary from time to time, but in real oxidation furnaces the fluctuations are observed to be tiny. The standard values of A and $\mathrm{B}$ under specific process conditions can be easily found, for example, in an IC process handbook. Eq. (4) can be rewritten as,

$$
\begin{aligned}
& a_{i}^{\prime}\left(A_{\text {std. }}+\delta A_{i}\right)+b_{i}^{\prime}\left(B_{\text {std. }}+\delta B_{i}\right)+c_{i}^{\prime}=0 \\
& \Rightarrow a_{i} \delta A_{i}+b_{i} \delta B_{i}+c_{i}=0 \quad i=1,2, \ldots n
\end{aligned}
$$

\begin{tabular}{|c|c|c|c|c|c|c|c|c|}
\hline \multicolumn{9}{|c|}{ Time: $16: 45$ Correlogram of Speed } \\
\hline Autocorre & lation & $\begin{array}{l}\text { Partial } \\
\text { Correlatio }\end{array}$ & & & $A C$ & PAC & Q-Stat & Prob \\
\hline & 1 & & & 1 & -0.204 & -0.204 & 1.3770 & 0.241 \\
\hline 14 & & 1 & & 2 & 0.231 & 0.198 & 3.2114 & 0.201 \\
\hline - & , & 10 & 1 & 3 & -0.218 & -0.152 & 4.9032 & 0.179 \\
\hline 18 & $i$ & 16 & i & 4 & 0.139 & 0.044 & 5.6203 & 0.229 \\
\hline 18 & , & 1 & 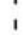 & 5 & -0.045 & 0.057 & 5.6982 & 0.337 \\
\hline$i$ & i & 16 & i & 6 & 0.145 & 0.090 & 6.5357 & 0.366 \\
\hline i & 1 & 15 & i & 7 & 0.024 & 0.091 & 6.5601 & 0.476 \\
\hline i & 1 & $i$ & i & 8 & 0.054 & 0.031 & 6.6895 & 0.570 \\
\hline i & i & $i$ & i & & -0.012 & 0.015 & 6.6958 & 0.669 \\
\hline 1 & i & 10 & $i$ & & -0.052 & -0.069 & 6.8241 & 0.742 \\
\hline & i & 1 & 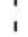 & & -0.140 & -0.184 & 7.8199 & 0.729 \\
\hline 14 & i & 18 & 1 & & -0.074 & -0.139 & 8.1122 & 0.776 \\
\hline 11 & 1 & 17 & 1 & & -0.007 & -0.031 & 8.1146 & 0.836 \\
\hline $1 d$ & 1 & 10 & i & & -0.068 & -0.110 & 8.3893 & 0.868 \\
\hline 10 & 1 & 1 & $i$ & & -0.094 & -0.154 & 8.9588 & 0.880 \\
\hline 11 & 1 & 17 & 1 & & 0.002 & 0.027 & 8.9590 & 0.915 \\
\hline
\end{tabular}

Fig. 4 Correlogram of $S_{1} \sim S_{30}$ 


\begin{tabular}{|c|c|c|c|c|c|c|}
\hline \multicolumn{7}{|c|}{$\begin{array}{l}\text { Correlogram of } \mathrm{D}(\text { Speed) } \\
\text { Time: } 16: 46\end{array}$} \\
\hline Autocorrelation & $\begin{array}{l}\text { Partial } \\
\text { Correlation }\end{array}$ & & $\mathrm{AC}$ & PAC & Q-Stat & Prob \\
\hline$\square 1$ & $\square$ & 1 & $1-0.669$ & -0.669 & 14.371 & 0.000 \\
\hline 1 & 10 & $i$ & 20.369 & -0.143 & 18.839 & 0.000 \\
\hline$\square-1$ & 1 & 1 & $3-0.345$ & -0.293 & 23.001 & 0.000 \\
\hline ו ו & 몀 & 1 & 0.208 & -0.264 & 24.560 & 0.000 \\
\hline 1 [ 1 & 믄 & 1 & -0.133 & -0.215 & 25.220 & 0.000 \\
\hline 171 & 믄 & 1 & 0.103 & -0.200 & 25.639 & 0.000 \\
\hline 1 & 18 & 1 & -0.040 & -0.133 & 25.704 & 0.001 \\
\hline 1 & 18 & 1 & 0.024 & -0.113 & 25.729 & 0.001 \\
\hline 1 & 11 & 1 & 90.008 & -0.029 & 25.732 & 0.002 \\
\hline 1 & 13 & 1 & 100.007 & 0.070 & 25.734 & 0.004 \\
\hline 10 & 1 & 1 & $11-0.062$ & -0.016 & 25.929 & 0.007 \\
\hline 1 & 1 단 & 1 & $12-0.012$ & -0.137 & 25.937 & 0.011 \\
\hline
\end{tabular}

Fig. 5 Correlogram of the first order differential sequence

where $A_{\text {std. }}$ and $B_{\text {std. }}$ represent the standard or the long term values of $\mathrm{A}$ and $\mathrm{B}, \delta A_{i}$ and $\delta B_{i}$ represent the fluctuations at time $i(i=1,2, \ldots, n)$.

3) A point, $\mathrm{P}\left(\delta A_{i}, \delta B_{i}\right)$, can easily be calculated with Eq. (5) and the criterion,

$\min .\left[\left(\delta A_{i}\right)^{2}+\left(\delta B_{i}\right)^{2}\right]$

Namely, $P$ is the point which is on line and nearest to the origin, as shown in Fig. 2.

4) As $\delta A_{i}, \delta B_{i}$ being calculated, we now have two time series. $\delta A_{n+1}$ and $\delta B_{n+1}$ for the next oxidation can be predicted, from $\delta A_{1} \sim \delta A_{n}$ and $\delta B_{1} \sim \delta B_{n}$, respectively.

5) Since the process status for the next oxidation is estimated, the processing time can be adjusted accordingly to achieve a more accurate process control.

6) The above steps can be generalized to deal with multidimension cases. For example, if $\tau$ in Eq. (3) is not a constant but a variable, then we face a 3-dimension prediction problem. By forecasting the next step $A, B$, and $\tau$, and correcting the processing time, the process can be similarly controlled.

\begin{tabular}{|c|c|c|c|c|c|c|c|c|}
\hline \multicolumn{9}{|c|}{$\begin{array}{l}\text { Correlogram of } \mathrm{D}(\text { Speed.2) } \\
\text { ime: } 16: 48\end{array}$} \\
\hline Autocorr & elation & $\begin{array}{l}\text { Partial } \\
\text { Correlatio }\end{array}$ & & & $A C$ & PAC & Q-Stat & Prob \\
\hline & 1 & & 1 & 1 & -0.809 & -0.809 & 20.338 & 0.000 \\
\hline 1 & $\square$ & ' & i & 2 & 0.541 & -0.327 & 29.782 & 0.000 \\
\hline$\square$ & 1 & 15 & 1 & 3 & -0.378 & -0.200 & 34.576 & 0.000 \\
\hline 1 & & 18 & 1 & 4 & 0.234 & -0.218 & 36.497 & 0.000 \\
\hline 10 & I & 15 & 1 & 5 & -0.129 & -0.123 & 37.102 & 0.000 \\
\hline 1 & 1 & 17 & 1 & 6 & 0.069 & -0.081 & 37.281 & 0.000 \\
\hline 1 & 1 & 1 & 1 & 7 & -0.026 & -0.035 & 37.309 & 0.000 \\
\hline 1 & 1 & 1 & 1 & 8 & -0.010 & -0.061 & 37.313 & 0.000 \\
\hline 1 & 1 & 1 & 1 & 9 & 0.026 & -0.046 & 37.343 & 0.000 \\
\hline 1 & 1 & 1 & 1 & & 0.007 & 0.099 & 37.345 & 0.000 \\
\hline 1 & 1 & $1 p$ & 1 & & -0.025 & 0.103 & 37.376 & 0.000 \\
\hline 1 & 1 & 14 & 1 & & -0.013 & -0.061 & 37.385 & 0.000 \\
\hline
\end{tabular}

Fig. 6 Correlogram of the second order differential sequence

\begin{tabular}{|c|c|c|c|c|}
\hline \multicolumn{5}{|c|}{ 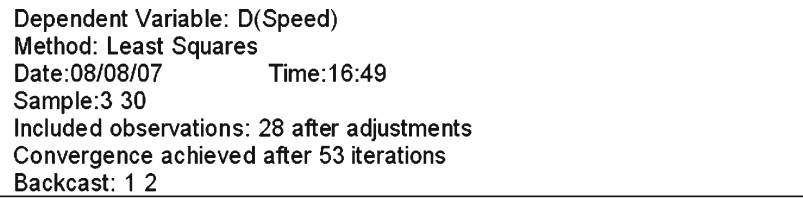 } \\
\hline Variable & Coefficient & Std. Error & $\mathrm{t}$-Statistic & Prob. \\
\hline $\begin{array}{l}\mathrm{C} \\
\mathrm{AR}(1) \\
\mathrm{MA}(1) \\
\mathrm{MA}(2)\end{array}$ & $\begin{array}{l}0.020027 \\
-0.864235 \\
-0.279197 \\
-0.670951 \\
\end{array}$ & $\begin{array}{l}0.018060 \\
0.275443 \\
0.395999 \\
0.354593 \\
\end{array}$ & $\begin{array}{r}1.108906 \\
-3.137614 \\
-0.705046 \\
-1.892171 \\
\end{array}$ & $\begin{array}{l}0.2785 \\
0.0045 \\
0.4876 \\
0.0706 \\
\end{array}$ \\
\hline $\begin{array}{l}\text { R-squared } \\
\text { Adjusted R-squared } \\
\text { S.E. of regression } \\
\text { Sum squared resid } \\
\text { Log likelihood } \\
\text { Durbin-Watson stat }\end{array}$ & $\begin{array}{l}0.640745 \\
0.595838 \\
0.801338 \\
15.41141 \\
-31.37093 \\
2.001714 \\
\end{array}$ & \multicolumn{2}{|c|}{$\begin{array}{l}\text { Mean dependent var } \\
\text { S.D. dependent var } \\
\text { Akaike info criterion } \\
\text { Schwarz criterion } \\
\text { F-statistic } \\
\text { Prob(F-statistic) } \\
\end{array}$} & $\begin{array}{l}0.021429 \\
1.260486 \\
2.526495 \\
2.716810 \\
14.26831 \\
0.000015 \\
\end{array}$ \\
\hline $\begin{array}{l}\text { Inverted AR Roots } \\
\text { Inverted MA Roots }\end{array}$ & $\begin{array}{r}-.86 \\
97\end{array}$ & & & \\
\hline
\end{tabular}

Fig. 7 Coefficients and other data in the $\operatorname{ARMA}(1,2)$ model

\section{An example on dynamic process control}

In this section, we show an example on dynamic control in a SiGe epitaxial process. In this example, thickness nonuniformity is decreased by tuning the processing time for each run. SiGe eptaxial layers were grown on wafers of different batches with a self-made UHV/CVD system, which is shown in Fig. 3.

Since the equipment was not designed for massive production, it is mainly used for research purpose. After each epitaxy process, the layer thickness was measured. Apparent variance can be found in the recorded thickness data. There are two ways to improve the tool performance. One is the hardware redesign and optimization, the other is to relief this issue by advanced process control. The second one is preferred here for economical consideration.

Thickness data from 60 continuous runs are studied to find out whether the dynamic process control can evidently improve the process uniformity. Here we use the method presented in Section 3.

1) Thickness data and processing times of the 60 runs have been recorded. These data, $D_{t}$ and $T_{t}(t$ from one to 60 ), are known.

\begin{tabular}{|c|c|c|c|c|c|c|c|c|}
\hline $\begin{array}{l}\text { Date:08/0 } \\
\text { Sample:3 } \\
\text { Included } \\
\text { Q-statistic }\end{array}$ & $\begin{array}{l}8 / 07 \\
30 \\
\text { bsen } \\
\text { prob }\end{array}$ & $\begin{array}{l}\text { me: } 16: 51 \\
\text { ns: } 28 \\
\text { ties adjuste }\end{array}$ & relogr & & term(s & & & \\
\hline Autocorrel & ation & Partial Cor & elation & & $A C$ & PAC & Q-Stat & Prob \\
\hline & I & 1 & 1 & 1 & -0.012 & -0.012 & 0.0044 & \\
\hline 1 & I & i & i & 2 & -0.026 & -0.026 & 0.0258 & \\
\hline 1 단 & I & 1 & i & 3 & -0.161 & -0.162 & 0.8956 & \\
\hline 17 & I & 13 & i & 4 & 0.087 & -0.095 & 1.1599 & 0.281 \\
\hline 1 & 1 & I & 1 & 5 & 0.040 & 0.028 & 1.2195 & 0.543 \\
\hline 1 & I & 1 & 1 & 6 & 0.037 & 0.008 & 1.2706 & 0.736 \\
\hline 1 & 1 & 1 & 1 & 7 & 0.073 & 0.048 & 1.4809 & 0.830 \\
\hline 1 & I & I & 1 & 8 & 0.006 & 0.014 & 1.4824 & 0.915 \\
\hline 1 & I & $\mathrm{I}$ & 1 & 9 & 0.013 & 0.032 & 1.4903 & 0.960 \\
\hline 10 & I & 10 & 1 & & -0.098 & -0.078 & 1.9408 & 0.963 \\
\hline 1 든 & 1 & 1므 & 1 & & -0.201 & -0.202 & 3.9418 & 0.862 \\
\hline 10 & 1 & 10 & 1 & & -0.107 & -0.133 & 4.5422 & 0.872 \\
\hline
\end{tabular}

Fig. 8 Correlogram of residuals of the $\operatorname{ARMA}(1,2)$ model 


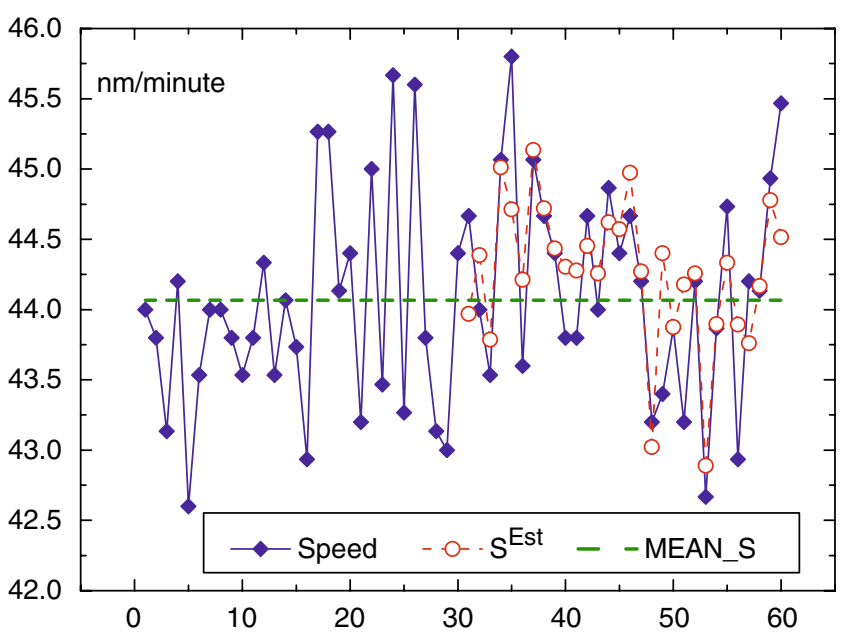

Fig. 9 Estimated speeds vs. real speeds

2) Eq. (2) is used as the process model. Then the growing speeds, $S_{t}$ ( $t$ from one to 60 ), are calculated.

$S_{t}=D_{t} / T_{t}$

3) $S_{1}, S_{2} \ldots S_{30}$ form a time series and $S_{31}$ can be estimated by ARMA model. Steps shown in Fig. 1 are followed to build an ARMA $(p, q)$ model.

Correlogram of the time series is shown in Fig. 4. The values are almost zero, which shows that the time series is stationary. It also shows that all the values of correlogram are within confidence interval, which means the data correlation is quite weak. Therefore higher order differential sequence should be further examined for model identification. Correlograms of the first and second order differential sequences of $\left\{S_{i}\right\}$ are shown in Figs. 5 and 6.

Both of the first and second order differential sequences are feasible for ARMA modeling. And the former is illustrated here as an example.

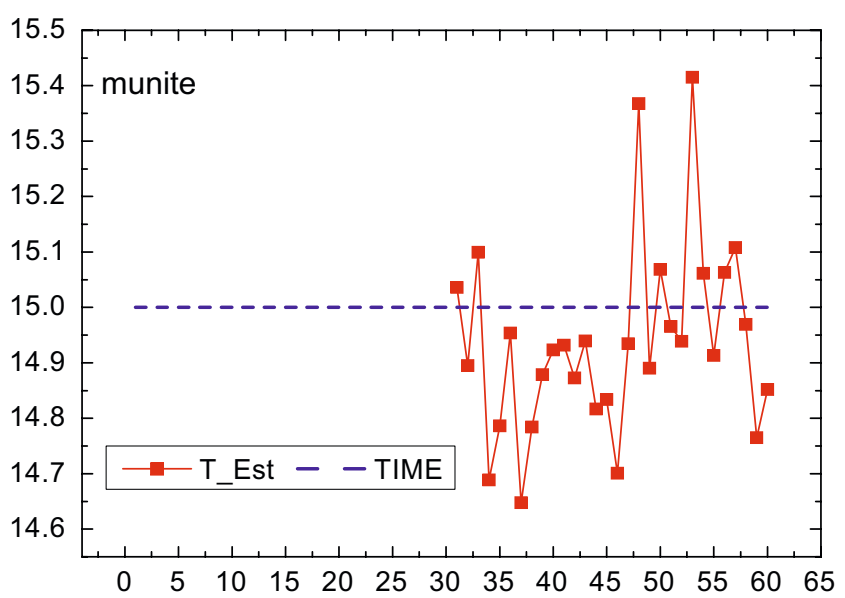

Fig. 10 Dynamic processing times vs. fixed processing times

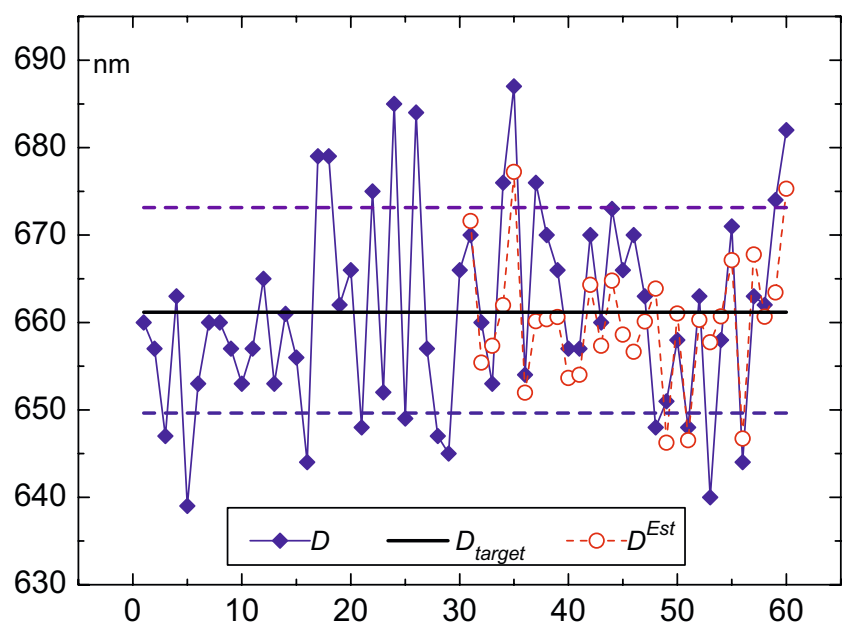

Fig. 11 Layer thickness after adjustment vs. original layer thickness

Figure 5 shows that the autocorrelation function goes into the confidence interval at the second order and long tailed, the partial autocorrelation function goes into the confidence interval at the first order and long tailed. Therefore we try an ARMA $(1,2)$ model. With the existing series $\left\{S_{i}\right\}$, the ARMA $(1,2)$ model is established. The coefficients are calculated and shown in Fig. 7.

After the modeling, it is necessary to make sure that the model is valid. The correlogram of the residuals is shown in Fig. 8, and that the residuals are observed to be white noise. So the ARMA $(1,2)$ model is self-consistent.

The 31st epitaxial speed $S_{31}{ }^{E s t}$ is then estimated by the ARMA model. Calculation shows that $S_{31}{ }^{E s t}=44.09849$ $(\mathrm{nm} / \mathrm{min})$.

4) The adjusted processing time $T_{31}{ }^{E s t}$ for the $31 s t$ epitaxial growing is calculated.

$$
T_{31}^{E s t}=D_{\text {target }} / S_{31}^{E s t}
$$

where $D_{\text {target }}$ is the thickness we want to get from the epitaxy process and in this work it is set to be the mean value of all observed thicknesses $(661 \mathrm{~nm})$.

Table 2 Results with and without dynamic process control

\begin{tabular}{lclc}
\hline & $D$ & $D_{\text {target }}$ & \multicolumn{1}{c}{$D^{\text {Est }}$} \\
\hline Mean & 663.0000 & 661.1500 & 660.0494 \\
Median & 663.0000 & 661.1500 & 660.3339 \\
Maximum & 687.0000 & 661.1500 & 677.2151 \\
Minimum & 640.0000 & 661.1500 & 646.2508 \\
Std. dev. & 11.11073 & 0.000000 & 7.314567 \\
Skewness & -0.015956 & NA & 0.169837 \\
Kurtosis & 2.599254 & NA & 3.313851 \\
Jarque-Bera & 0.202020 & NA & 0.267352 \\
Probability & 0.903924 & NA & 0.874874 \\
Sum & 19890.00 & 19834.50 & 19801.48 \\
Sum sq. dev. & 3580.000 & 0.000000 & 1551.584 \\
Observations & 30 & 30 & 30 \\
\hline
\end{tabular}


Since the real speed for the $31 s t$ epitaxial growing is known (we have calculated all the growing speeds, $S_{t}(t$ from 1 to 60)), the conclusion can be reached that if dynamic processing time, $T_{31}{ }^{E s t}$, is adopted, the epitaxial layer thickness must be $T_{31}{ }^{E s t} \times S_{31}\left(=D_{31}{ }^{E s t}\right)$. This value is compared with the tested thickness, $D_{31}$, to see if the predictionadjustment scheme works better.

5) $S_{32}$ can also be predicted with the known epitaxial speeds $S_{2} \sim S_{31}$. If the processing time is adjusted in the same way, a layer thickness, $D_{32}{ }^{E s t}$, will be calculated and compared with the measured experiment result $D_{32}$.

Using the method described above, $D_{31}{ }^{E s t} \sim D_{60}{ }^{E s t}$ are all obtained. And we find the ARMA $(1,2)$ model is feasible for all these calculations. The estimated thickness sequence can be used to compare with the real thickness sequence, $D_{31} \sim D_{60}$.

6) In Fig. 9, the forecasted epitaxial layer growing speeds is shown together with the real speed. The results exhibit close trends match with acceptable value tolerance.

The comparison of the dynamic processing times and the fixed processing time in experiment is shown in Fig. 10, and the adjusted layer thickness $D^{E s t}$ and the real thickness $D$ are shown in Fig. 11, it sees the thicknesses are closer to the mean value after the adjustment.

Some statistics are listed in Table 2, which shows the thickness fluctuation is successfully reduced by our prediction-adjustment scheme. We can see that if the processing times are dynamically adjusted, the standard deviation (std. dev.) and the sum of deviation square (sum sq. dev.) of epitaxial thickness can be evidently decreased. If we define that the epitaxy thickness within $661 \pm 12 \mathrm{~nm}$ is eligible to use, shown as the region confined by the two lines in Fig. 11, then the yield of the original process is $66.7 \%$ (20 out of 30 points fall in the range), while the yield becomes $83.3 \%$ if dynamic process control is applied ( 25 out of 30 points fall in the region).

\section{Conclusion}

It is very difficult to deal with the process fluctuation due to its stochastic properties. Such fluctuations become more and more important when the technology node goes smaller and smaller. To further improve IC product yields, process fluctuations must be reduced.

Time series analysis is a powerful statistical tool and becomes an important way to provide IC yield enhancement when applied to IC industry. However, it can not be directly used for process control. By separating variables, one or more undisturbed time series could be obtained for accurate forecasting. Then the processing time can be adjusted accordingly to reduce the process fluctuations. Our example shows that the uniformity of the epitaxial process step can be improved by more than $10 \%$ on the self-made epitaxy system by using the self-reactive adjustment method.

The dynamic process control method we introduced here can easily be applied to a variety of IC processes other than epitaxial process we discussed.

The current rule in IC process control prefers fixed process parameters. Our work indicates that higher yield can be achieved by introducing dynamic process parameters.

Open Access This article is distributed under the terms of the Creative Commons Attribution Noncommercial License which permits any noncommercial use, distribution, and reproduction in any medium, provided the original author(s) and source are credited.

\section{References}

1. Cunningham SP, Spanos CJ, Voros K (1995) Semiconductor yield improvement: results and best practices. IEEE Trans Semicond Manuf 8:103-109 doi:10.1109/66.382273

2. Bergeret F, Le Gall C (2003) Yield improvement using statistical analysis of process dates. IEEE Trans Semicond Manuf 16:535542 doi:10.1109/TSM.2003.815204

3. Gardner RM, Bieker J, Elwell S (2000) Solving tough semiconductor manufacturing problems using data mining. IEEE/SEMI Advanced Semiconductor Manufacturing Conference, pp 46-55

4. Chen WC, Tseng SS, Hsiao KR, Liu CC (2004) A data mining projects for solving low-yield situations of semiconductor manufacturing. IEEE/SEMI Advanced Semiconductor Manufacturing Conference, pp 129-134

5. Wilcox R, Forhan T, Starkey G (1998) Design for manufacturability: a key to semiconductor manufacturing excellence. IEEE/SEMI Advanced Semiconductor Manufacturing Conference, pp 308-313

6. Zorian Y (2004) Optimizing manufacturability by design for yield. IEEE/SEMI International Electronics Manufacturing Technology Symposium, pp 255-258

7. White KP, Trybula WJ, Athay RN (1997) Design for semiconductor manufacturing - perspective. IEEE Trans Compon Packag Manuf Technol Part B Adv Packag 20:58-72 doi:10.1109/3476.585145

8. Naguib H (1993) The implementation of total quality management (TQM) in a semiconductor manufacturing operation. IEEE Trans Semicond Manuf 6:156-162 doi:10.1109/66.216934

9. Hwang YD (2006) The practices of integrating manufacturing execution system and six sigma methodology. Int J Adv Manuf Technol 30:761-768 doi:10.1007/s00170-005-0090-1

10. Fieler PE, Loverro N (1991) Defects tail off with six-sigma manufacturing. IEEE Circuits Devices Mag 7:18-20 doi:10.1109/ 101.86129

11. Nixon P, Conway J, Maimon J (1993) Statistical process control and the drive for six sigma in a VLSI Pilot Line. IEEE/CHMT International Electronic Manufacturing Technology Symposium, pp 219-228

12. Tseng SC, Chen SL, Chang JK (2004) An effective recipe control and management system (RCMS) deployed in semiconductor manufacturing. Semiconductor Manufacturing Technology Workshop Proceedings, pp 135-138 
13. Ozelkan EC, Cakanyildirim M (2006) Test wafer management for semiconductor manufacturing. IEEE Trans Semicond Manuf 19:241-251 doi:10.1109/TSM.2006.873401

14. Wu MC, Chien CS, Lu KS (2006) Yield improvement planning for the recycle processes of test wafers. Int J Adv Manuf Technol 27:1228-1234 doi:10.1007/s00170-004-2316-Z

15. Lin CT, Chang CW, Chen CB (2006) A simple approach to solving multi-response quality characteristic problems in CMOS ion implantation. Int J Adv Manuf Technol 28:592-595 doi:10.1007/ s00170-004-2396-9

16. Chung S-H, Pearn WL, Kang H-Y (2005) A linear programming model for the control wafers downgrading problem. Int $\mathrm{J}$ Adv Manuf Technol 25:377-384 doi:10.1007/s00170-003-1854-0

17. Card JP, Sniderman DL, Klimasauskas C (1997) Dynamic neural control for a plasma etch process. IEEE Trans Neural Netw 8:883-901 doi:10.1109/72.595886
18. Himmel CD, Kim TS, Krauss A, Kamen EW, May GS (1995) Real-time predictive control of semiconductor manufacturing processes using neural networks. Proc Am Contr Conf 2:12401244 doi:10.1109/ACC.1995.520948

19. Alvi NS (1995) Manufacturing process improvements using advanced control methodologies. Proc Japan Int Electr Manuf Technol Symp, pp 276-282

20. McDonald CJ (1997) Copy EXACTLY! a paradigm shift in technology transfer method. IEEE/SEMI Advanced Semiconductor Manufacturing Conference, pp 414-417

21. Box GEP, Jenkins GM, Reinsel GC (1994) Time series analysis: forecasting and control. Prentice-Hall, New Jersey

22. Pandit SM, Wu SM (1983) Time series and system analysis, with applications. Wiley, New York

23. Deal BE, Grove AS (1965) General relationship for the thermal oxidation of silicon. J Appl Phys 36:3770-3778 doi:10.1063/1.1713945 